\title{
Brief Research Report: Bayesian versus REML Estimations with Noninformative Priors in Multilevel Single-Case Data
}

\begin{abstract}
Recently, researchers have used multilevel models for assessing intervention effects in singlecase studies, which are based on the replication of interrupted time-series designs across a small number of cases. Researchers estimating these multilevel models have primarily relied on restricted maximum likelihood (REML) techniques, but Bayesian approaches have also been suggested. The purpose of this Monte Carlo simulation study was to examine the impact of estimation method (REML versus Bayesian with noninformative priors) on the estimation of treatment effects (relative bias, root mean square error) and on the inferences about those effects (interval coverage) for autocorrelated single-case data. Simulated conditions varied with regard to the number of participants, series length, and the distribution of the variance within and across cases. REML and Bayesian estimation led to unbiased estimates of the fixed effects, but differentially impacted the inferences about the fixed effects and the estimates of the variances. Implications for applied single-case researchers and methodologists are discussed.
\end{abstract}

Keywords: single-case research, multilevel modeling, restricted maximum likelihood, Bayesian estimation, estimation method, noninformative priors 
Single-case research measures an outcome for a single case, or a few cases, over time to examine the effectiveness of treatments (Kazdin, 2011). There is a growing interest in singlecase designs due to the many advantages that they offer. For example, single-case designs allow researchers to investigate the treatment effect for each individual as well as individual variations in the treatment effect, which information is difficult to capture using group comparison designs (Barlow, Nock, \& Hersen, 2009). Another advantage of single-case studies is that they allow researchers to study populations that have a low prevalence rate (e.g., children with autism, another example?) that are difficult to study with large sample based designs (Van den Noortgate \& Onghena, 2003a).

One of the main features of single-case designs is that data are obtained before implementing intervention (baseline phase) and after implementing intervention (treatment phase). AB design is the most basic design that has a baseline phase and a treatment phase. The additional designs include an extension of this design, such as an $\mathrm{ABAB}$ design that has more phases for removal of the treatment and reintroduction of the treatment. Multiple-baseline designs are another alternative designs that are commonly used. In these designs, the treatment is introduced to different cases at different points in time so that the initiation of the treatment phase can be staggered across time for the different cases. Multiple-baseline design have several benefits in that the treatment effects can be more likely to be attributed to the treatment, not to extraneous events (e.g., history or maturation), and they can be used to study multiple cases at the same time (Ferron \& Rendina-Gobioff, 2005; Kazdin, 2011 ; Shadish \& Sullivan, 2011). Visual examples of $\mathrm{AB}, \mathrm{ABAB}$, and multiple baseline designs are provided in the additional document. 
Multilevel modeling (MLM) has been suggested as an alternative method for analyzing single-case data (Shadish, Kyse, \& Rindskopf, 2013; Shadish \& Rindskopf, 2007; Van den Noortgate \& Onghena, 2003a, 2003b). In single-case analysis, multilevel modeling can provide in-depth information about treatment effects, such as individual treatment effect, average treatment effect, and the variability of treatment effects across cases. The MLM approach has the flexibility needed to handle various methodological issues that may arise with single-case studies, such as the need to model possible dependency in the errors, linear or nonlinear trends, and count outcomes (Hembry, Bunuan, Beretvas, Ferron, \& Van den Noortgate, in press; Shadish, Kyse, \& Rindskopf, 2013; Van den Noortgate \& Onghena, 2003a).

There are several estimation methods available to run MLM analyses. The most commonly used method for single-case studies is restricted maximum likelihood (REML, e.g., Ferron, Bell, Hess, Rendina-Gobioff, \& Hibbard, 2009; Ferron, Farmer, \& Owens, 2010; Van Noortgate \& Onghena, 2003a), because like full ML the estimation algorithms are readily available, but REML has advantages over full ML when sample sizes are small. However, it has been found that technical issues (i.e., nonconvergence) can arise when REML is used to estimate more complicated models (Baek, Petit-Bois, \& Ferron, 2012) and that variance estimates can be substantially biased in the small sample sizes typical of single-case studies (Ferron, Bell, Hess, Rendina-Gobioff, \& Hibbard, 2009). Bayesian estimation (Lindley \& Smith, 1972) is another method that can potentially offer more benefits for estimating complicated models (Gelman, Carlin, Stern, \& Rubin, 2004; Shadish, Kyse, \& Rindskopf, 2013). For example, Baek, Petit-Bois and Ferron (2013) found that estimation of a more complicated single-case model that allows between case variation in the level-1 error structure which required to estimate more parameters 
than traditional single-case model had failed to converge previously when using REML could be estimated using Bayesian methods.

In order to choose the most appropriate estimation method, it is critical for researchers to know the effects of the different methods on parameter estimates and the associated statistical inferences. Despite the importance of this issue, little is known about whether the use of these different estimation methods with MLM analyses of single-case data yields similar estimates of and inferences about treatment effects. Moeyaert, Rindskopf, Onghena and Van den Noortgate (2017) compared the impact of Maximum likelihood (ML) estimation (Full ML and REML) and Bayesian estimation in an initial study using single-case data. They found Bayesian estimation results were similar to ML results for fixed effects, and although the variance estimates were less biased with Bayesian estimation than ML, the bias was still substantial. Their study was limited to an examination of weakly informative priors for variances and to models where it was assumed there was no autocorrelation in the data.

The choice of priors can substantially impact inferences, but suggestions in the literature for selecting priors for variance components are inconsistent . Therefore, it is important to examine a variety of feasible priors when comparing ML and Bayesian estimation. Many researchers have suggested various noninformative prior distributions for the variance parameters in multilevel models, including uniform, inverse-gamma family, and half-t distributions (Berger \& Strawderman, 1996; Daniels \& Kass, 1999; Gelman, 2006). For example, Gelman (2006) demonstrated the impact of various proposed noninformative prior distributions for the level-2 variance parameters in multilevel models. He found that the uniform distribution generally works well in that it has little impact on posterior inferences, as long as the number of level-2 units is at least equal to 3 which is required to ensure a proper posterior 
density. Although Moeyaert and her colleagues (2017) found that both ML and Bayesian estimation procedures resulted in biased and imprecise variance estimates when the number of participants is smaller (i.e., 3) under weakly informative priors, performance under noninformative priors, which are typically the first choice of applied researchers, have not been studied. Therefore, it is worthwhile to extend the study of Bayesian methods to include the choice of noninformative priors.

In addition, in single-case studies, the errors are typically considered to be autocorrelated as opposed to independent (e.g., Matyas \& Greenwood, 1997). It has been well documented that flawed inferences can arise if the dependency of errors is not taken into account in the statistical model. Specifically, for multilevel models, many researchers have found that when level-1 errors are incorrectly assumed to be independent, this may bias estimation of the standard errors of the fixed effects and estimation of the random effects' variance components (Ferron, Dailey, \& Yi, 2002; Ferron, Bell, Hess, Rendina-Gobioff, \& Hibbard, 2009; Kwok, West, \& Green, 2007; Sivo, Fan \& Witta, 2005; Sivo \& Willson, 2000). Therefore, it is important to extend the comparison of estimation methods for single-case data with autocorrelated errors since the study from Moeyaert and her colleagues (2017) examined only data with no autocorrelated error as previously mentioned.

This study was conducted to extend the examination of the degree to which multilevel modeling estimates and inferences from single-case data are impacted when using different estimation methods. Specifically we extended the examination to include autocorrelated data and the comparison of REML to Bayesian estimation with noninformative priors.

\section{REML estimation in single-case design}

Several methodological studies on the use of REML with multilevel models for singlecase data have found unbiased estimates of the fixed effects, correct inferences (e.g., controlled 
Type I error rates, accurate confidence intervals) for fixed effects when using either the Kenward-Roger or Satterthwaite approach to adjust standard errors and degrees of freedom (Kenward \& Roger, 1997, 2009; Satterthwaite,1941), but biased variance component estimates (Ferron, Bell, Hess, Rendina-Gobioff, \& Hibbard, 2009; Moeyaert, Ugille, Ferron, Beretvas, \& Van den Noortgate, 2013; Owens \& Ferron, 2012).

A basic two-level multilevel model for single-case studies with two phases is shown in Equations 1 and 2. Equation 1 refers to the first level of the multilevel model, and describes the variation of scores within cases. Index $i$ stands for the measurement occasion $(i=0,1, . . I)$ and $j$ for the case $(j=0,1, \ldots J)$. A dummy coded variable, Phase $_{i j}$, is an indicator of a baseline and treatment phase, and Time $_{i j}$ variable is an indicator of a linear trend.

$$
\begin{gathered}
y_{i j}=\beta_{0 j}+\beta_{1 j} \text { Time }_{i j}+\beta_{2 j} \text { Phase }_{i j}+\beta_{3 j} \text { Phase }_{i j} * \text { Time }_{i j} \\
+e_{i j} \quad \text { and } e_{i j} \sim N\left(0, \Sigma_{e}\right)
\end{gathered}
$$

More specifically, $y_{i j}$ is the observed value (outcome) at the $i^{\text {th }}$ observation for the $j^{\text {th }}$ case. $\beta_{0 j}$ is the baseline intercept for the $j^{\text {th }}$ case and Phase $_{i j}$ is a dichotomous variable that indicates the phase in which the observation occurred, where 0 indicates the baseline phase and 1 indicates the treatment phase. $\beta_{1 j}$ is the difference between the baseline level and the treatment level (shift in level) for the $j^{\text {th }}$ case when Time $_{i j}$ is equal to 0. Time $_{i j}$ is an indicator of time elapsed during the study (e.g., days or session number) and thus $\beta_{2 j}$ is the baseline slope for the $i^{\text {th }}$ case, $\beta_{3 j}$ is the change in slopes between the baseline phase and the treatment phase (shift in slope), and $e_{i j}$ is the residual that indicates within case variation (level-1 errors) and is assumed to be multivariate normally distributed $\mathrm{N}\left(0, \Sigma_{e}\right)$. Various covariance matrices of $\Sigma_{e}$ can be modeled in single-case design. $\Sigma_{e}$ can be modeled as being autocorrelated (e.g., unstructured, compound symmetry, banded toeplitz or moving average, first-order autoregressive $[\mathrm{AR}(1)])$ or as independent $\left(\sigma^{2} \mathrm{I}\right)$ 
(Goldstein, 1995; Goldstein, Healy, \& Rasbash, 1994; Heitjan \& Sharma, 1997; Jennrich \& Schluchter, 1986; Ware, 1985; Wolfinger, 1993; Yang \& Goldstein, 1996). The most commonly assumed dependent error structure for single-case analyses is the first order autoregressive model (Ferron et al., 2009).

Equation 2 describes at the second level, which models variability across cases in the baseline intercept, the baseline slope, the shift in level, and the shift in slope.

$$
\left\{\begin{array}{l}
\beta_{0 j}=\theta_{00}+u_{0 j} \\
\beta_{1 j}=\theta_{10}+u_{1 j} \\
\beta_{2 j}=\theta_{20}+u_{2 j} \\
\beta_{3 j}=\theta_{30}+u_{3 j}
\end{array} \text { with }\left[\begin{array}{l}
u_{0 j} \\
u_{1 j} \\
u_{2 j} \\
u_{3 j}
\end{array}\right] \sim N\left(0, \Sigma_{u}\right)\right.
$$

$\theta_{00}$ is the average baseline intercept and $\theta_{10}$ is the average shift in level at Time $i j$ equal to $0, \theta_{20}$ is the average baseline slope, and $\theta_{30}$ is the average shift in slope. $u_{0 j}, u_{1 j}, u_{2 j}$ and $u_{3 j}$ are level-2 errors and are assumed to be multivariate normally distributed $\mathrm{N}\left(0, \Sigma_{u}\right)$ and $\Sigma_{u}$ is typically assumed to be a diagonal matrix in single-case applications.

\section{Bayesian estimation in multilevel single-case data analysis}

Bayesian estimation provides an alternative estimation method for addressing some of the technical challenges encountered when using likelihood-based estimation procedures in multilevel analysis (Gelman, Carlin, Stern, \& Rubin, 2004; Shadish, Rindskopf, \& Hedges, 2008; Shadish \& Rindskopf, 2007). Studies in multilevel analyses have found that Bayesian methods perform as well or better than likelihood methods regarding bias, efficiency, and interval coverage (Browne, 2008; Baldwin \& Fellingham, 2013; Moeyaert, Rindskopf, Onghena, \& Van den Noorgate, 2017). For multilevel single-case research, the Bayesian approach could 
provide more accurate results when using small samples or unequal sample sizes per subject (Shadish, Kyse, \& Rindskopf, 2013) or when REML suffers from convergence issues (Baek, Petit-bois, \& Ferron, 2013). Bayesian methods are feasible in computationally intensive scenarios through use of Markov Chain Monte Carlo (MCMC) procedures (e.g., Chen \& Shao, 1999; Cowles \&Carlin, 1996; Gelman, Carlin, Stern, \& Rubin, 2004; Gilks, Richardson, \& Spiegelhalter, 1996; Tierney, 1994).

Because Bayesian estimation is implemented using a probability framework, the multilevel model can also be expressed using probability distributions. For instance, assuming a first-order autoregressive model for the errors at level-1, the two-level single-case model which is shown in Equations 1 and 2 can be re-written as seen in Equation 3:

$$
\begin{aligned}
& y_{\mathrm{ij}} \sim \operatorname{Normal}\left(\mu_{\mathrm{ij},} \sigma_{\mathrm{e}}^{2}\right) \\
& \mu_{\mathrm{ij}}=\beta_{0 \mathrm{j}}+\beta_{1 \mathrm{j}} \text { Time }_{i j}+\beta_{2 \mathrm{j}} \text { Phase }_{i j}+\beta_{3 \mathrm{j}} \text { Time }_{i j} * \text { Phase }_{i j} \\
& \alpha_{0 \mathrm{j}}=\mu_{0 \mathrm{j}} \\
& \alpha_{\mathrm{ij}}=\mu_{\mathrm{ij}}+\rho\left(y_{(\mathrm{i}-1) \mathrm{j}}-\mu_{(\mathrm{i}-1) \mathrm{j}}\right) \quad(i \geq 1) \\
& \beta_{0 \mathrm{j}} \sim \operatorname{Normal}\left(\theta_{00}, \sigma_{u_{0}}^{2}\right) \\
& \beta_{1 \mathrm{j}} \sim \operatorname{Normal}\left(\theta_{10}, \sigma_{u_{1}}^{2}\right) \\
& \beta_{2 \mathrm{j}} \sim \operatorname{Normal}\left(\theta_{20}, \sigma_{u_{2}}^{2}\right) \\
& \beta_{3 \mathrm{j}} \sim \operatorname{Normal}\left(\theta_{30}, \sigma_{u_{3}}^{2}\right)
\end{aligned}
$$

where $y_{i j}$ is the observed value for the $j^{\text {th }}$ case at the $i^{\text {th }}$ point in time, and has a normal distribution as a prior distribution with the mean of $\alpha_{\mathrm{ij}}$ and variance of $\sigma^{2}{ }_{\mathrm{e}} ; \alpha_{\mathrm{ij}}$ is defined by adding the correlated error term between the adjacent two time points to $\mu_{\mathrm{ij}}$, where $\rho$ represents the autocorrelation and $\left(y_{(\mathrm{i}-1) \mathrm{j}}-\mu_{(\mathrm{i}-1) \mathrm{j}}\right)$ represents the error term at the $i-1$ time point. When $i=0$, $\alpha_{0 \mathrm{j}}$ is equal to $\mu_{0 \mathrm{j}}$. In the Bayesian framework, the average fixed effect and variance parameters $\left(\theta_{00}, \theta_{10}, \theta_{20}, \theta_{30}, \sigma_{u_{0}}^{2}, \sigma_{u_{1}}^{2}, \sigma_{u_{2}}^{2}\right.$ and $\left.\sigma_{u_{3}}^{2}\right)$ are referred to as hyperparameters in that they are priors 
on the prior parameters (i.e., $\beta_{0 \mathrm{j},} \beta_{1 \mathrm{j}}, \beta_{2 \mathrm{j}}, \beta_{3 \mathrm{j}}$ ) of the model. In addition, it is assumed, here, that each regression coefficient, $\beta_{0 \mathrm{j}}, \beta_{1 \mathrm{j}}, \beta_{2 \mathrm{j}}, \beta_{3 \mathrm{j}}$, follows a normal distribution. Prior knowledge about the hyperparameters can be taken into account by stating a prior distribution about these parameters, while a lack of prior knowledge is reflected in the use of noninformative prior distributions (e.g., a normal distribution with a very large variance). In a Bayesian analysis, these prior distributions are combined with the observed data in order to obtain posterior distributions for the parameters of interest.

\section{Simulation}

A Monte Carlo simulation study was conducted that examined conditions that varied in series length, number of cases, and the distribution of the variance across levels in the design. The series length, or level-1 sample size was set to either 10 or 30 , based on surveys of single-case applications (Ferron et al, 2010; Shadish \& Sullivan, 2011), where 10 was found to be a relatively short series for single-case studies and 30 found to be a moderate series length. The number of cases was also varied such that the second level sample size was either 4 or 8 , where 4 is a typical number of cases, and 8 is a relatively large number of cases for single-case studies (Ferron et al, 2010; Shadish \& Sullivan, 2011).

The level-1 error covariance structure $\left(\Sigma_{e}\right)$ was generated to follow a first-order autoregressive error structure $[\mathrm{AR}(1)]$, with a variance parameter of 1.0 and an autocorrelation value of .2, which is typical of the level of autocorrelation observed in single-case studies (Shadish \& Sullivan, 2011). The first-order autoregressive error structure was selected because it is the most commonly assumed autocorrelated error structures for single-case data analysis. The level-2 error variance in intercept, shift in level, baseline slope, and shift in slope were also varied to reflect the differences observed across multilevel analyses of single-case data. Some 
analyses have found that there is more variance at level-1 (Ferron et al., 2010; Moeyaert et al., 2013), whereas other analyses have found more variance at level-2 (Moeyaert et al., 2013). More specifically, the first condition of the level-2 error variances in intercept, phase, time, and interaction had the values of $.5, .5, .05$, and .05 , respectively, such that each level-2 error variance has half variance of the level-1 (1.0). The second condition of the level-2 error variance in intercept, phase, time, and interaction had values of 2, 2, .2, and .2 respectively, such that each level-2 error variance has twice variance of the level-1 (1.0).

Data sets were generated using a multiple-baseline design. When there were four cases and the series length was 10 , the number of baseline observations for the four cases were $3,4,6$, and 7, respectively. The number of baseline observations per case was selected based on a general rule of a minimum number of observations (three) for a baseline phase (Barlow, Nock, \& Hersen, 2009). The number of baseline observations was set to a minimum number of 3 for the first case and then gradually increased for the second, third and fourth case to create a multiplebaseline design in which the initiation of the treatment phase was staggered across time for the different cases. When the series length was 30, the number of baseline observations for the four cases were 9, 13, 17, and 21, respectively. For conditions with eight participants, the baseline lengths were the same as for conditions with four participants, but there were two participants assigned to each of the baseline lengths.

Equations 1 and 2 were used to generate 1000 data sets for each of the data conditions obtained by crossing series length, number of cases, and the amount of variance at level-2. All simulated data sets were analyzed using both REML and Bayesian estimation. For each analysis, Time $_{i j}$ for the shift in slope $\left(\right.$ Time $_{i j} *$ Phase $_{i j}$ ) was centered so that 0 corresponded to the first observation of the treatment phase. For REML estimation, the two-level model (i.e., Equations 1 
and 2) was estimated using PROC MIXED in SAS version 9.3 (SAS Institute Inc., 2008). It was assumed $\Sigma_{e}$ was first-order autoregressive, $\Sigma_{u}$ was diagonal, and the Kenward-Roger method was used to estimate standard errors and calculate the degrees of freedom (Ferron et al, 2009; Owens \& Ferron, 2012).

For Bayesian estimation, the model in Equation 3 was estimated via WinBUGS software version 1.4.3 which uses a Gibbs sampler. A common prior distribution used in the multilevel models for the fixed effect parameters is a noninformative normal distribution with a mean of 0 and a variance of $10^{6}$, and for $\sigma$ a uniform distribution with the lower limit of 0 and the upper limit of 100 (Gelman, Carlin, Stern, \& Rubin, 2004; Gelman, 2006). Thus, these prior distributions were used for the fixed effects (i.e., $\left.\theta_{00}, \theta_{10}, \theta_{20}, \theta_{30}\right)$ and standard deviation unit of level-1 error $(\sigma)$, respectively, in this study.

$$
\begin{aligned}
& \theta_{00}, \theta_{10}, \theta_{20}, \theta_{30} \sim \operatorname{Normal}\left(0,1000^{2}\right) \\
& \sigma \sim \operatorname{Uniform}(0,100)
\end{aligned}
$$

The value of 100 used for the upper bound of the prior for the level- 1 error standard deviation is considered as sufficiently noninformative because the true value of $\sigma$ will be generated to be a value of one in this study. The lower limit of $\sigma$ is set to 0 due to the fact that the value of the standard deviation cannot be negative. In addition, uniform distributions were used as priors for the level-2 error variance parameters (i.e., $\sigma_{u_{0}}^{2}, \sigma_{u_{1}}^{2}, \sigma_{u_{2}}^{2}$ and $\sigma_{u_{3}}^{2}$ ) matching Gelman's (2006) recommendation. Unlike for the fixed effects and level-1 error variance, noninformative prior distributions for level-2 variance parameters have been more difficult to construct. As previously mentioned, Gelman (2006) has found that the uniform distribution generally works well for the level-2 error variance in that it has little impact on posterior inferences, as long as the number of level-1 units is at least equal to 3. By his recommendation, a noninformative uniform prior was 
selected for the standard deviation of the level-2 errors. Specifically, the noninformative prior distributions for the standard deviation unit of the level-2 errors were assigned to be the uniform distribution with the lower limit of 0 and an upper limit of 100 in this study.

$$
\sigma_{u_{0}}, \sigma_{u_{1}}, \sigma_{u_{2}}, \sigma_{u_{3}} \sim \text { Uniform }(0,100)
$$

For autocorrelation, $\rho$, a reasonable noninformative prior distribution can be a normal distribution. Shadish and Sullivan (2011) summarize the characteristics of single-case designs using 809 published studies. In their report, the histogram of the autocorrelation estimates among the published studies followed an approximately normal distribution ranging from -.931 to .786 . Thus, we could construct the noninformative prior for $\rho$ that follows a normal distribution with a mean of 0 and a standard deviation $(\sigma)$ of 1000 . However, since $\rho$ is a correlation parameter, the scale of this parameter should be the same as a correlation scale, from -1 to 1 . Therefore, the scale of the prior distribution for $\rho$ should be stationary restricted so that its range falls between 1 and 1 (Gamerman \& Lopes, 2006).

$$
\rho \sim \operatorname{Normal}\left(0,1000^{2}\right) \mathrm{I}(-1<\rho<1)
$$

Random samples of simulated datasets (one sample for each condition) were first generated to test convergence and to make decisions about the number of iterations, and the burn-in period. The various diagnostic criteria such as trace plots, history plots, Kernel density plots, and Brooks-Gelman-Rubin (BGR) plots were used in monitoring convergence. After checking all simulation data sets for convergence, it was decided to use a burn-in of 2,000 iterations and to run an additional 60,000 iterations to form the posterior distribution.

To compare estimation methods, parameter bias and RMSE were examined for the fixed effects and variance components. For the fixed effects, interval coverage was also examined, specifically $95 \%$ confidence interval coverage for REML and 95\% credible interval coverage for 
Bayesian estimation. Relative bias for parameters whose known value is anything other than 0 was also computed which can be represented as a percentage of the known parameter value. According to Hoogland and Boomsma (1998) criteria, parameter estimate is considered as unbiased if the relative bias is smaller than the $5 \%$. Mean of the posterior distribution was used for all point estimates. The relationship between design factors and outcome measures were then evaluated using factorial ANOVA with eta-squared values $\left(\eta^{2}\right)$. Models were built to find medium effects or larger (eta-squared values $\left(\eta^{2}\right)$ were equal to or greater than .06). The computed $\eta^{2}$ values were interpreted using Cohen's (1988) standards with a small effect size as .01 ; a medium effect as .06; and a large effect as .14 or greater.

\section{Results}

The average values for bias, RMSE, and CI coverage across conditions were discussed in the result section. The detailed results of each simulated conditions for the fixed effects and the variance components are provided in Tables 1 and 2, respectively in appendix.

\section{Fixed Treatment Effects}

The average relative bias of the fixed treatment effect estimates is minimal (close to 0) across all parameters, ranging between - .006 and .001 and differences across the two methods are also minimal. The smallest average relative biases were found in the shift in level parameter, $M=.001, S D=.02$ for Bayesian estimation, and $M=.002, S D=.02$ for REML estimation. In addition, none of the design factors (series length, number of cases, and variation in the level-2 error) show a substantial impact on the average relative bias estimates.

However, the average interval coverage rates tend to be overly conservative when using the Bayesian estimation method (average coverage estimates for shift in level and shift in slope parameters of .984 and .983 , respectively). Average coverage rates for the REML estimation 
method were closer to the nominal level (average coverage estimates of.947 and .950 , for shift in level and shift in slope, respectively).

The relationship between interval coverage for the fixed effects and the design factors was also explored and it was found that the 2-way interaction between the number of cases and the estimation methods has a medium effect $\left(\eta^{2}=.13\right)$ on interval coverage. The difference in coverage between the estimation methods decreases when the number of cases increases.

The average RMSE values for the fixed effects parameters are very similar across the estimation methods $(M=.67, S D=.19$ for Bayesian estimation of shift in level, and $M=.68, S D$ $=.20$ for REML estimation of shift in level; and the $M=.22, S D=.09$ for both Bayesian and REML estimation of shift in slope)._Further examination showed that the number of cases and the variation in the level- 2 error have a medium effect $\left(\eta^{2}=.08, \eta^{2}=.09\right.$, respectively) on the average RMSE values for the fixed effects. As the number of cases is increased, the average values of RMSE for the all fixed effects are decreased, ranging from .064 to .248. In addition, for lower true values for the variation in the level-2 error, the average values of RMSE for the fixed effects are lower, ranging from .076 to .241 .

\section{Variance components}

The REML method using PROC MIXED originally estimates all between-case and within-case parameters in variances, while the Bayesian method is specified to estimate all between-case and within-case parameters as standard deviations. As a consequence, the two methods produce estimation results on different scales. In order to make the estimates comparable, the square root was taken of all variance estimates and these standard deviations (SD) are reported. 
The level-2 SD estimates are positively biased when using Bayesian estimation and negatively biased when using REML estimation. The average relative biases for all level-2 error SDs were ranging from .64 to 1.0 in Bayesian estimation, while ranging from -.04 to -.17 in REML estimation with the variation in estimates substantially explained by the estimation method $\left(\eta^{2}=.49\right)$. Unlike other SD components, the estimate of the level-1 error SD was positively biased regardless of the estimation method, $M=.02$ for the both estimation methods. The estimate of the autocorrelation parameter is positively biased $(M=.08)$ when using Bayesian estimation, and negatively biased $(M=-.12)$ when using REML estimation.

Further examination found that the two-way interaction between the number of cases and the estimation methods has a large effect $\left(\eta^{2}=.20\right)$ on the average relative bias estimates for the level-2 error SD components. The difference in the estimation methods is decreased when the number of cases is increased. Further examination of the level-1 error SD and the autocorrelation also indicated that the two-way interaction between the series length per case and the estimation method has a medium effect $\left(\eta^{2}=.11\right)$ and has a large effect $\left(\eta^{2}=.22\right)$ on the relative bias in the level-1 error SD and autocorrelation estimate, respectively. As the series length increases, the difference in bias between methods is reduced for both parameters.

The average RMSE value for the level-2 errors' SD is different across the estimation methods. For the shift in level parameters, the average RMSE value is bigger for Bayesian estimation(1.22) than REML estimation(.68), while for the shift in slope, the average RMSE value is smaller for Bayesian estimation (.57) than REML estimation (.80). For level-1 error SD and autocorrelation, the average RMSE value is bigger for REML estimation $(.14, .17$, respectively) than Bayesian estimation (.10, .16, respectively). 
Further examination showed that the amount of variation in the level-2 error has a large effect $\left(\eta^{2}=.20\right)$ on the average RMSE values for the level-2 error SD. As the variation in the level-2 error is decreased, the average values of RMSE for the level-2 error SD are decreased, which is consistent with the impact on the average RMSE values for the fixed effects. In addition, for the intercept and shift in level parameters, the 2-way interaction between the number of cases and the estimation methods has a large effect $\left(\eta^{2}=.19\right)$ which indicates the difference in the estimation methods is decreased as the number of cases is increased. This interaction effect was not observed for the average RMSE values for the baseline slope and the shift in slope parameters. For the level-1 SD and the autocorrelation parameters, it was found that the series length per case has a large effect $\left(\eta^{2}=.64\right)$. As the series length per case is increased, the average RMSE values are decreased.

\section{Discussion}

The purpose of this study was to examine the degree to which multilevel modeling estimates and inferences were impacted by using different estimation methods, specifically REML and Bayesian with noninformative priors, when analyzing autocorrelated multiplebaseline data. Similar to the previous study using informative priors, the results indicated that the fixed effects are not biased for either estimation method. Unlike the previous study using informative priors that found similar results between REML and Bayesian estimation, the interval coverage for the fixed effects tended to be overly conservative when using the Bayesian approach with noninformative priors.

For the level-2 error SD, similar to the previous research using informative priors, it was found that as the number of cases increased, the relative bias decreased, regardless of the estimation method. This indicates that the number of cases is a critical factor to make more precise estimates, regardless of the estimation method and the choice of priors. For the estimate 
of the autocorrelation and level-1 error parameter, it was found that the difference of the bias estimates between the two estimation methods decreased as the series length per case increased. These results imply that although estimation methods have little to no impact on the estimates of the fixed effects, there is some impact on the inferences about fixed effects, and more substantial impact on the estimates of the variance components.

As with other simulation studies, this study is limited by the conditions simulated. Although the conditions were selected to be typical of single-case studies, and to complement those of the study by Moeyaert et al. (2017), they represent only a subset of the conditions that may be encountered by single-case researchers. Therefore, the conclusions from this study are limited to the selected conditions. Future research that covers a larger range of the possible conditions (e.g., nonlinear trends, heterogeneous variance structures) is needed to make more general conclusions about the impact of the estimation methods on multilevel modeling estimates and inferences in the context of single-case studies.

Across the studied conditions we studied, as well as across the conditions studied by Moeyaert et al. (2017), both methods appear to offer a reasonable approach for estimating and testing the average treatment effect parameters from single-case data, and thus both can be recommended for that purpose. Some researchers, however, are interested not only in the average treatment effects, but also in how much the effects vary across participants. The variance components were biased under both forms of estimation, therefore future statistical work is necessary to develop alternative methods of estimation aimed at reducing these biases. One estimation alternative that could be explored is bootstrapping, which is a computer intensive resampling approach that has been discussed in the context of more conventional applications of multilevel modeling (Meijer, Van der Leeden, \& Busing, 1995). Another alternative to be 
developed and explored is the addition of more strongly informative priors into the Bayesian framework. Our study purposefully focused on relatively noninformative priors because these are commonly used by applied researchers, and Moeyaert et al. (2017) focused on weakly informative priors, but more work is needed to determine reasonable informative priors for error variances in the context of single-case research, that in turn could possibly improve Bayesian estimates. 


\section{Acknowledgement}

This research is funded by the Institute of Education Sciences, U.S. Department of

Education, Grant number R305D110024. The opinions expressed are those of the authors and do not represent views of the Institute or the U.S. Department of Education. 


\section{References}

Baek, E., Petit-Bois, M., \& Ferron, M. J. (April, 2012). The effect of error structure specification on the meta-analysis of single-case studies of reading fluency interventions. Paper presented at the annual meeting of the American Educational Research Association, Vancouver, BC, Canada.

Baek, E., Petit-Bois, M., \& Ferron, M. J. (April, 2013). A Feasible Way to Vary Level-1 Error Structure Across Participants in Multilevel Models for Single-Case Data. Paper presented at the annual meeting of the American Educational Research Association, San Francisco, CA.

Barlow, D. H., Nock, M. K., \& Hersen, M. (2009). Single Case Experimental Designs: Strategies for Studying Behavior Change (3rd ed.). Boston, MA: Pearson.

Baldwin, S.A. \& Fellingham, G.W. (2013). Bayesian methods for the analysis of small sample multilevel data with a complex variance structure. Psychological Methods, 18, 151-164.

Berger, J. O., \& Strawderman, W. E. (1996). Choice of hierarchical priors: Admissibility in estimation of normal means. Annals of Statistics, 24, 931-951.

Browne, W. (2008). MCMC estimation in MLwinN. Bristol, England: Centre for Multilevel Modeling.

Chen, M. H., \& Shao, Q. M. (1999). Monte Carlo estimation of Bayesian credible and HPD Intervals. Journal of Computational and Graphical Statistics, 8, 69-92.

Cohen, J. (1988). Statistical power analysis for the behavioral sciences. New York: Lawrence Erlbaum Associates.

Cowles, M. K., \& Carlin, B. P. (1996). Markov chain Monte Carlo convergence diagnostics: A comparative review. Journal of the American Statistical Association, 91, 883-904. 
Daniels, M.J. \& Kass, R.E. (1999). Nonconjugate Bayesian estimation of covariance matrices and its use in hierarchical models. Journal of the American Statistical Association, 94, $1254-1263$.

Ferron, J., Farmer, J., \& Owens, C. (2010). Estimating individual treatment effects from multiple-baseline data: A Monte Carlo study of multilevel modeling approaches. Behavior Research Methods, 42, 930-943.

Ferron, J. M., Bell, B. A., Hess, M. R., Rendina-Gobioff, G., \& Hibbard, S. T. (2009). Making treatment effect inferences from multiple-baseline data: The utility of multilevel modeling approaches. Behavior Research Methods, 41, 372-384.

Gamerman, D., \& Lopes, H.(2006). Markov chain Monte Carlo: Stochastic simulation for Bayesian inference, second edition. Boca Raton, FL: Chapman and Hall/CRC.

Gelman, A., Carlin, J.B., Stern, H.S., \& Rubin, D.B. (2004). Bayesian data analysis (2nd Ed). Boca Raton, FL: Chapman and Hall/CRC.

Gelman, A. (2006). Prior distributions for variance parameters in hierarchical models. Bayesian Analysis, 1, 515-533.

Gilks, W.R., Richardson, S., \& Spiegelhalter, D.J. (1996). Markov chain Monte Carlo in practice. Boca Raton, FL: Chapman and Hall/CRC.

Goldstein, H., \& Yang, M. (2000). Meta-analysis using multilevel models with an application to the study of class size effects. Journal of the Royal Statistical Society, Series C: Applied Statistics, 49, 339-412.

Hembry, I., Bunuan, R., Beretvas, S. N., \& Ferron, J. M., Van den Noortgate, W. (in press). Estimation of a nonlinear intervention phase trajectory for multiple baseline design data. Journal of Experimental Education. 
Hoogland, J. J., \& Boomsma, A. (1998). Robustness studies in covariance structure modeling. An overview and a meta-analysis. Sociological Methods \& Research, 26, 329 -367.

Hox, J. J., \& de Leeuw, E. D. (1997). Meta-analyse via multiniveau-modellen. Tijdschrift Voor Onderwijsresearch, 22, 16-28.

Kazdin, A. E. (2011). Single-Case Research Designs: Methods for Clinical and Applied Settings (2nd ed.). New York: Oxford University Press.

Kenward, M. G. \& Roger, J. H. (1997). Small sample inference for fixed effects from restricted maximum likelihood. Biometrics, 53, $983-997$.

Kenward, M. G. \& Roger, J. H. (2009). An improved approximation to the precision of fixed effects from restricted maximum likelihood. Computational Statistics and Data Analysis, $53,2583-2595$.

Lindley, D.V., \& Smith, A.EM. (1972). Bayes estimates for the linear model. Journal of the Royal Statistical Society, Series B, 34, 1-41.

Matyas, T. A. \& Greenwood, K. M. (1997). Serial dependency in single-case time series. In R. D. Franklin, D. B. Allison, \& B. S. Gorman (Eds.), Design and analysis of single-case research (pp. 215-243). Mahwah, NJ: Lawrence Erlbaum.

Meijer, E., van der Leeden, R., \& Busing, F. M. T. A. (1995). Implementing the bootstrap for multilevel models. Multilevel Modelling Newsletter, 7(2), 7-11.

Moeyaert, M., Ugille, M., Ferron, J., Beretvas, T., \& Van den Noortgate, W. (2014). Three-level analysis of single-case experimental data: Empirical validation. The Journal of Experimental Education, 82, 1-21. 
Moeyaert, M., Rindskopf, D., Onghena, P., \& Van den Noortgate, W. (2017). Multilevel modeling of single-case data: A comparison of Maximum likelihood and Bayesian estimation. Psychological Methods, 22, 760-778.

Owens, C. M., \& Ferron, J. M. (2012). Synthesizing single-case studies: A Monte Carlo examination of a three-level meta-analytic model. Behavior Research Methods, 44, 795805.

Patterson, H. D., \& Thompson, R. (1971). Recovery of inter-block information when block sizes are unequal. Biometrika, 58, 545-554.

Satterthwaite, F. E. (1941). Synthesis of variance. Psychometrika, 6, 309-316.

Shadish, W. R., Kyse, E. N., \& Rindskopf, D. M. (2013). Analyzing data from single-case designs using multilevel models: new applications and some agenda items for future research. Psychological Methods, 18, 385-405.

Shadish, W. R., \& Rindskopf, D. M. (2007). Methods for evidence based practice: Quantitative synthesis of single-subject designs. New Directions for Evaluation, 113, 95-109.

Shadish, W.R., Rindskopf, D. M., \& Hedges, L.V. (2008). The state of science in the metaanalysis of single-case experimental designs. Evidence-Based Communication Assessment and Intervention, 3, 188-196.

Shadish, W. R., \& Sullivan, K. J. (2011). Characteristics of single-case designs used to assess intervention effects in 2008. Behavior Research Methods, 43, 971-980.

Tierney, L. (1994). Markov chains for exploring posterior distributions. Annals of Statistics, 22, 1701-1762.

Van den Noortgate, W., \& Onghena, P. (2003a). Combining single-case experimental data using hierarchical linear models. School Psychology Quarterly, 18, 325-346. 
Van den Noortgate, W., \& Onghena, P. (2003b). Hierarchical linear models for the quantitative integration of effect sizes in single-case research. Behavior Research Methods, Instruments, \& Computers, 35, 1-10. 